\title{
Full discretization of stochastic Burgers equation with correlated noise
}

\author{
Dirk Blömker ${ }^{\mathrm{a}}$, Minoo Kamrani ${ }^{\mathrm{b}}$, S. Mohammad Hosseini ${ }^{\mathrm{b}}$ \\ Institut für Mathematik, Universität Augsburg, 86135 Augsburg, Germany ${ }^{a}$ \\ Department of Applied Mathematics, Tarbiat Modares University, \\ P. O. Box 14115-175, Tehran, Iran
}

September 11, 2012

\begin{abstract}
The main purpose of this paper is to investigate the spectral Galerkin method for spatial discretization. We combine it with the method introduced by Kloeden, Jentzen \& Winkel in [15] for temporal discretization of stochastic partial differential equations and study pathwise convergence. We consider the case of colored noise, instead of the usual space-time white noise that was used before for the spatial discretization. The rate of convergence in uniform topology is estimated for the stochastic Burgers equation. Numerical examples illustrate the estimated convergence rate.
\end{abstract}

Keywords: stochastic partial differential equations, colored noise, Galerkin approximation, stochastic Burgers equation.

\section{Introduction}

In this article the numerical approximation of nonlinear parabolic stochastic partial differential equations (SPDEs) is considered. Following the ideas of Blömker \& Jentzen [3] for the case of space-time white noise, a numerical method for simulating nonlinear SPDEs with additive noise for the case of colored noise is proposed and analyzed. The main novelty in this article is to estimate the spatial and temporal discretization error in the $L^{\infty}$-topology in the case of colored noise. This is different from the usual space-time white noise, that was considered before in [3] for spatial discretization.

We consider as forcing term an infinite dimensional stochastic process expanded in the eigenfunctions of the linear operator $A$ present in the SPDE. We focus on the case where the Brownian motions are not independent. This is due to the fact that the spatial covariance operator of the forcing does not commute with $A$.

In order to illustrate the main result of this article we consider stochastic Burgers equation with Dirichlet boundary conditions on a bounded domain. To 
be more precise, let $T>0,(\Omega, \mathcal{F}, \mathbb{P})$ be a probability space, and let the spacetime continuous stochastic process $X:[0, T] \times \Omega \rightarrow C([0,1], \mathbb{R})$ be the unique solution of the SPDE

$$
d X_{t}=\left[\frac{\partial^{2}}{\partial x^{2}} X_{t}-X_{t} \cdot \frac{\partial}{\partial x} X_{t}\right] d t+d W_{t}, \quad X_{t}(0)=X_{t}(1)=0, \quad X_{0}=0,
$$

for $t \in[0, T]$ and $x \in(0,1)$. The noise is given by a cylindrical Wiener process $W_{t}, t \in[0, T]$ defined later.

There are numerous publications for colored or correlated noise of the type presented here, which is white in time and colored in space. For Burgers equation see $[2,5,4,8]$, for example. Here we refrain from the usual assumption, that the covariance of $W$ and the Laplacian are jointly diagonal.

The existence and uniqueness of solutions of the stochastic Burgers equation was studied by Da Prato \& Gatarek in [5] for colored noise. Da Prato \& Zabzcyk in [6] and [7] studied (1) for space-time white noise and Gyöngy \& Nualart in [9] studied the equation the whole real line.

Alabert and Gyöngy obtained a spatial discretization of this equation in $L^{2}$-topology [1]. Recently, Blömker and Jentzen [3] obtained a bound on spatial discretization error in uniform topology by the spectral Galerkin method for the case of space-time white noise.

The spectral Galerkin method has been extensively studied for stochastic partial differential equations with space-time white noise. See for example [13, $16,17,18,19]$.

Hausenblas investigated the discretization error of semilinear stochastic evolution equations in $L^{p}$-spaces, Banach spaces and quasi linear evolution equations driven by nuclear or space time white noise in [11, 12]. Gyöngy and Shardlow in $[21,10]$ apply finite differences in order to approximate the mild solution of parabolic SPDEs driven by space-time white noise. Yoo investigates the mild solution of parabolic SPDEs by finite differences in [22].

Our aim here is to extend the result of [3]. First we discuss the case of colored noise not diagonal with respect to the eigenfunctions of the Laplacian. Secondly, using the time discretization that was introduced in [15], we obtain an error estimate for the full space-time discretization.

The reminder of this paper is organized as follows. Section 2 gives the setting and the assumptions. In Section 3 we investigate spatial discretization error, and in Section 4 the temporal error is obtained. Finally, in the last section numerical examples are presented.

\section{Setting and assumptions}

Fix $T>0$, let $(\Omega, \mathcal{F}, \mathbb{P})$ be a probability space and both $\left(V,\|\cdot\|_{V}\right)$ and $\left(W,\|\cdot\|_{W}\right)$ be $\mathbb{R}$-Banach spaces. Moreover, let $P_{N}: V \rightarrow V, N \in \mathbb{N}$, be a sequence of bounded linear operators.

Throughout this article the following assumptions will be used.

Assumption 1. Let $S:(0, T] \rightarrow L(W, V)$ be a continuous mapping satisfying

$$
\sup _{0<t \leq T}\left(t^{\alpha}\left\|S_{t}\right\|_{L(W, V)}\right)<\infty, \quad \sup _{N \in \mathbb{N}} \sup _{0 \leq t \leq T}\left(t^{\alpha} N^{\gamma}\left\|S_{t}-P_{N} S_{t}\right\|_{L(W, V)}\right)<\infty,
$$

where $\alpha \in[0,1)$ and $\gamma \in(0, \infty)$ are given constants. 
Assumption 2. Let $F: V \rightarrow W$ be a locally Lischitz continuous mapping, which satisfies

$$
\sup _{\|v\|_{V},\|w\|_{V} \leq r} \frac{\|F(v)-F(w)\|_{W}}{\|v-w\|_{V}}<\infty
$$

for every $r>0$.

Assumption 3. Let $O:[0, T] \times \Omega \rightarrow V$ be a stochastic process with continuous sample paths and

$$
\sup _{N \in \mathbb{N}} \sup _{0 \leq t \leq T} N^{\gamma}\left\|O_{t}(\omega)-P_{N}\left(O_{t}(\omega)\right)\right\|_{V}<\infty
$$

for every $\omega \in \Omega$, where $\gamma \in(0, \infty)$ is given in Assumption 1 .

Assumption 4. Let $X^{N}:[0, T] \times \Omega \rightarrow V, \quad N \in \mathbb{N}$, be a sequence of stochastic processes with continuous sample paths such that

$$
\sup _{M \in \mathbb{N}} \sup _{0 \leq s \leq T}\left\|X_{s}^{M}(\omega)\right\|_{V}<\infty
$$

and

$$
X_{t}^{N}(\omega)=\int_{0}^{t} P_{N} S_{t-s} F\left(X_{s}^{N}(\omega)\right) d s+P_{N}\left(O_{t}(\omega)\right),
$$

for every $t \in[0, T], \omega \in \Omega$ and every $N \in \mathbb{N}$.

Blömker and Jentzen [3] obtained the following Theorem.

Theorem 1. Let Assumptions 1-4 be fulfilled. Then, there exists a unique stochastic process $X:[0, T] \times \Omega \rightarrow V$ with continuous sample paths, which fulfills

$$
X_{t}(\omega)=\int_{0}^{t} S_{t-s} F\left(X_{s}(\omega)\right) d s+O_{t}(\omega),
$$

for every $t \in[0, T]$ and every $\omega \in \Omega$. Moreover, there exists a $\mathcal{F} / \mathcal{B}([0, \infty))$ measurable mapping $C:[0, \infty) \rightarrow \Omega$ such that

$$
\sup _{0 \leq t \leq T}\left\|X_{t}(\omega)-X_{t}^{N}(\omega)\right\|_{V} \leq C(\omega) \cdot N^{-\gamma},
$$

holds for every $N \in \mathbb{N}$ and every $\omega \in \Omega$, where $\gamma \in(0, \infty)$ is given in Assumption 1 .

\section{Spatial discretization for the case of colored noise}

Now we will show that Assumptions 1-4 are satisfied for Burgers equation in the case of colored noise. Therefore from Theorem 1 we can conclude convergence of the Galerkin method for this equation. Most of the results are already proven in [3]. We only state the results needed later in the proofs, and the modifications necessary due to the presence of colored noise.

In the reminder of the paper define $V=C^{0}([0,1]), W=H^{-1}(0,1)$. The mapping $\partial: V \rightarrow W$ is given by

$$
(\partial v)(\varphi)=\left(v^{\prime}\right)(\varphi):=-<v, \varphi^{\prime}>_{L_{2}}=-\int_{0}^{1} v(x) \varphi^{\prime}(x) d x
$$

for every $v \in V$ and $\varphi \in H^{1}(0,1)$, is a bounded linear mapping from $V$ to $W$.

From Lemma 4.6 and 4.8 in [3] we have the following Lemmas. 
Lemma 2. The mapping $S:(0, T] \rightarrow L\left(H^{-1}(0,1), C^{0}([0,1])\right)$ given by

$$
\left(S_{t}(w)\right)(x)=\sum_{n=1}^{N}\left(2 \cdot e^{-n^{2} \pi^{2} t} \cdot w(\sin (n \pi(.))) \cdot \sin (n \pi x)\right)
$$

for every $x \in[0,1], w \in H^{-1}(0,1)$ and every $t \in(0, T]$, is well defined and satisfies Assumption 1.

From Assumption 1 we derive

$$
\sup _{0<t \leq T}\left(t^{\alpha}\left\|S_{t} \partial\right\|_{L(V, V)}\right)<\infty
$$

where $\alpha$ was introduced in Assumption 1.

Remark 1 As we can see from Lemma 4.6 in [3], Assumption 1 is satisfied for $\alpha=\frac{3}{4}$, and $\gamma \in\left[0, \frac{1}{2}\right)$.

Lemma 3. The mapping $F: C^{0}([0,1]) \rightarrow H^{-1}(0,1)$, given for every $v \in$ $C^{0}([0,1])$ by $F(v)=\partial_{x}\left(v^{2}\right)$, satisfies Assumption 2.

In the following we present details on the $Q$-Wiener process $W$ corresponding to the colored noise, in order to prove Assumption 3 later. We focus on a $d$-dimensional setting, while the result needed later is for $d=1$. Let $\beta^{i}$ : $[0, T] \times \Omega \rightarrow \mathbb{R}, i \in \mathbb{N}^{d}$, be a family of Brownian motions that are not necessarily independent. They are correlated as given by

$\mathbb{E}\left(\beta^{k}(t) \beta^{l}(t)\right)=<Q e_{k}, e_{l}>\cdot t, \quad k=\left(k_{1}, \ldots, k_{d}\right) \in \mathbb{N}^{d}, t>0, l=\left(l_{1}, \ldots, l_{d}\right) \in \mathbb{N}^{d}$, where for every $k \in \mathbb{N}^{d}$

$$
e_{k}:[0,1]^{d} \rightarrow \mathbb{R}, \quad e_{k}(x)=2^{\frac{d}{2}} \sin \left(k_{1} \pi x_{1}\right) \cdot \ldots \cdot \sin \left(k_{d} \pi x_{d}\right), \quad x \in[0,1]^{d},
$$

are smooth functions. Furthermore, $Q$ is a symmetric non-negative operator, such that

$$
<Q e_{k}, e_{l}>=\int_{0}^{1} \int_{0}^{1} e_{k}(x) e_{l}(y) q(x-y) d y d x,
$$

for $k, l \in \mathbb{N}^{d}$ and some positive definite function $q$.

Moreover, for every $k \in \mathbb{N}^{d}$ define the real numbers $\lambda_{k}=\pi^{2}\left(k_{1}^{2}+\ldots+k_{d}^{2}\right) \in \mathbb{R}$.

Lemma 4. Assume there exists a $\rho>0$ such that in dimension $d \in\{1,2,3\}$

$$
\sum_{i \in N^{d}} \sum_{j \in N^{d}}\|i\|_{2}^{\rho-1}\|j\|_{2}^{\rho-1}\left|\left\langle Q e_{i}, e_{j}\right\rangle\right|<\infty
$$

Then there exists a stochastic process $O:[0, T] \times \Omega \rightarrow V$, which satisfies

$$
\begin{array}{r}
\sup _{0 \leq t_{1} \leq t_{2} \leq T} \frac{\left\|O_{t_{2}}(\omega)-O_{t_{1}}(\omega)\right\|_{V}}{\left(t_{2}-t_{1}\right)^{\theta}}<\infty, \\
\sup _{N \in \mathbb{N}} \sup _{0 \leq t \leq T} N^{\gamma}\left\|O_{t}(\omega)-P_{N}\left(O_{t}(\omega)\right)\right\|_{V}<\infty,
\end{array}
$$

for every $\omega \in \Omega$, every $\theta \in\left(0, \min \left\{\frac{1}{2}, \frac{\rho}{2}\right\}\right)$, every $\gamma \in(0, \rho)$. Furthermore, $O$ satisfies

$$
\mathbb{P}\left[\lim _{N \rightarrow \infty} \sup _{0 \leq t \leq T}\left\|O_{t}-\sum_{i \in\{1, \ldots, N\}^{d}}\left(-\lambda_{i} \int_{0}^{t} e^{-\lambda_{i}(t-s)} \beta_{s}^{i} d s+\beta_{t}^{i}\right) e_{i}\right\|_{V}=0\right]=1
$$


$\sup _{N \in \mathbb{N}}\left[\left(\mathbb{E}\left[\sup _{0 \leq t \leq T}\left\|O_{t}-P_{N} O_{t}\right\|_{V}^{p}\right]\right)^{\frac{1}{p}} N^{\gamma}\right]+\sup _{0 \leq t_{1} \leq t_{2} \leq T} \frac{\left(\mathbb{E}\left[\left\|O_{t_{2}}-O_{t_{1}}\right\|_{V}^{p}\right]\right)^{\frac{1}{p}}}{\left(t_{2}-t_{1}\right)^{\theta}}<\infty$ for every $p \in[1, \infty)$, and every $\gamma \in(0, \rho)$.

We need some technical Lemmas first, in order to prove this Lemma.

Lemma 5. For every $t_{1}, t_{2} \in[0, T]$ with $t_{1} \leq t_{2}$, and every $r \in(0,1)$ we have

$$
\begin{aligned}
& \mathbb{E}\left(\int_{0}^{t_{2}} e^{-\lambda_{i}\left(t_{2}-s\right)} d \beta_{s}^{i}-\int_{0}^{t_{1}} e^{-\lambda_{i}\left(t_{1}-s\right)} d \beta_{s}^{i}\right)\left(\int_{0}^{t_{2}} e^{-\lambda_{j}\left(t_{2}-s\right)} d \beta_{s}^{j}-\int_{0}^{t_{1}} e^{-\lambda_{j}\left(t_{1}-s\right)} d \beta_{s}^{j}\right) \\
& \quad \leq 2\left(\lambda_{i}+\lambda_{j}\right)^{r-1}\left(t_{2}-t_{1}\right)^{r}\left\langle Q e_{i}, e_{j}\right\rangle
\end{aligned}
$$

for all $i, j \in \mathbb{N}^{d}$.

Proof. Fix $t_{1}, t_{2} \in[0, T]$ with $t_{1} \leq t_{2}$, and $i, j \in \mathbb{N}^{d}$. Define $\Delta t=t_{2}-t_{1}$ and $\Lambda_{i j}=\lambda_{i}+\lambda_{j}$. We obtain

$$
\begin{aligned}
& \mathbb{E}\left(\int_{0}^{t_{2}} e^{-\lambda_{i}\left(t_{2}-s\right)} d \beta_{s}^{i}-\int_{0}^{t_{1}} e^{-\lambda_{i}\left(t_{1}-s\right)} d \beta_{s}^{i}\right) \cdot\left(\int_{0}^{t_{2}} e^{-\lambda_{j}\left(t_{2}-s\right)} d \beta_{s}^{j}-\int_{0}^{t_{1}} e^{-\lambda_{j}\left(t_{1}-s\right)} d \beta_{s}^{j}\right) \\
& =\mathbb{E}\left(\int_{t_{1}}^{t_{2}} e^{-\lambda_{i}\left(t_{2}-s\right)} d \beta_{s}^{i}+\left(e^{-\lambda_{i} \Delta t}-1\right) \int_{0}^{t_{1}} e^{-\lambda_{i}\left(t_{1}-s\right)} d \beta_{s}^{i}\right) \\
& \quad \times\left(\int_{t_{1}}^{t_{2}} e^{-\lambda_{j}\left(t_{2}-s\right)} d \beta_{s}^{j}+\left(e^{-\lambda_{j} \Delta t}-1\right) \int_{0}^{t_{1}} e^{-\lambda_{j}\left(t_{1}-s\right)} d \beta_{s}^{j}\right) \\
& =\int_{0}^{\Delta t} e^{-\Lambda_{i j} s}\left\langle Q e_{i}, e_{j}\right\rangle d s \\
& \quad+\left(e^{-\Lambda_{i j} \Delta t}-e^{-\lambda_{i} \Delta t}-e^{-\lambda_{j} \Delta t}+1\right) \cdot\left\langle Q e_{i}, e_{j}\right\rangle \cdot \frac{1-e^{-\Lambda_{i j} t_{1}}}{\Lambda_{i j}} \\
& =\left(1-e^{-\Lambda_{i j} \Delta t}+\left(e^{-\Lambda_{i j} \Delta t}-e^{-\lambda_{i} \Delta t}-e^{-\lambda_{j} \Delta t}+1\right)\left(1-e^{-\Lambda_{i j} t_{1}}\right)\right) \cdot \frac{\left\langle Q e_{i}, e_{j}\right\rangle}{\Lambda_{i j}} \\
& \leq\left(1-e^{-\Lambda_{i j} \Delta t}+\left(1-e^{-\Lambda_{i j} \Delta t}\right)\left(1-e^{-\Lambda_{i j} t_{1}}\right)\right) \cdot \frac{\left\langle Q e_{i}, e_{j}\right\rangle}{\Lambda_{i j}} \\
& \leq 2 \cdot \frac{1-e^{-\Lambda_{i j} \Delta t}}{\Lambda_{i j}} \cdot\left\langle Q e_{i}, e_{j}\right\rangle .
\end{aligned}
$$

Therefore, for every $r \in(0,1)$ we derive

$$
\begin{aligned}
& \mathbb{E}\left(\int_{0}^{t_{2}} e^{-\lambda_{i}\left(t_{2}-s\right)} d \beta_{s}^{i}-\int_{0}^{t_{1}} e^{-\lambda_{i}\left(t_{1}-s\right)} d \beta_{s}^{i}\right) \cdot\left(\int_{0}^{t_{2}} e^{-\lambda_{j}\left(t_{2}-s\right)} d \beta_{s}^{j}-\int_{0}^{t_{1}} e^{-\lambda_{j}\left(t_{1}-s\right)} d \beta_{s}^{j}\right) \\
& \leq 2 \cdot\left(\sup _{x>0} \frac{1}{x}\left(1-e^{-x}\right)\right)^{r} \cdot \Lambda_{i j}^{r-1}(\Delta t)^{r} \cdot\left|\left\langle Q e_{i}, e_{j}\right\rangle\right| \\
& =2 \cdot \Lambda_{i j}^{r-1}(\Delta t)^{r} \cdot\left|\left\langle Q e_{i}, e_{j}\right\rangle\right| .
\end{aligned}
$$


Lemma 6. For every $t_{1}, t_{2} \in[0, T]$, with $t_{1} \leq t_{2}, N \in \mathbb{N}, p \in[1, \infty)$ and every $\alpha, \theta \in\left(0, \frac{1}{2}\right]$ we have

$$
\begin{aligned}
& \left(\mathbb{E}\left[\sup _{x \in[0,1]^{d}}\left|O_{t_{2}}^{N}(x)-O_{t_{1}}^{N}(x)\right|^{p}\right]\right)^{\frac{1}{p}} \\
& \quad \leq C \sum_{i, j \in I_{N}}\|i\|_{2}^{2 \theta+2 \alpha-1}\|j\|_{2}^{2 \theta+2 \alpha-1}\left|\left\langle Q e_{i}, e_{j}\right\rangle\right|\left(t_{2}-t_{1}\right)^{\theta}
\end{aligned}
$$

where $C=C(d, p, \alpha, \theta)$ is a constant depending only on $d, p, \alpha$ and $\theta$. The stochastic process $O^{N}:[0, T] \times \Omega \rightarrow C\left([0,1]^{d}\right)$ is given by

$$
O_{t}^{N}=\sum_{i \in I_{N}} \int_{0}^{t} e^{-\lambda_{i}(t-s)} d \beta_{s}^{i} \cdot e_{i}
$$

for every $t \in[0, T]$ and every $N \in \mathbb{N}$, where $I_{N}=\{1, \ldots, N\}^{d}$.

Proof. Consider first

$$
\begin{aligned}
& \left(O_{t_{2}}^{N}(x)-O_{t_{1}}^{N}(x)\right)-\left(O_{t_{2}}^{N}(y)-O_{t_{1}}^{N}(y)\right) \\
& =\sum_{i \in I_{N}}\left(\int_{0}^{t_{2}} e^{-\lambda_{i}\left(t_{2}-s\right)} d \beta_{s}^{i}-\int_{0}^{t_{1}} e^{-\lambda_{i}\left(t_{1}-s\right)} d \beta_{s}^{i}\right) \cdot\left(e_{i}(x)-e_{i}(y)\right),
\end{aligned}
$$

$\mathbb{P}$-a.s. for every $x, y \in[0,1]^{d}$. Hence, expanding the square of the series as a double sum and using Lemma 5 we obtain (again with $\Delta t=t_{2}-t_{1}$ and $\left.\Lambda_{i j}=\lambda_{i}+\lambda_{j}\right)$

$$
\begin{aligned}
& \mathbb{E}\left|\left(O_{t_{2}}^{N}(x)-O_{t_{1}}^{N}(x)\right)-\left(O_{t_{2}}^{N}(y)-O_{t_{1}}^{N}(y)\right)\right|^{2} \\
& \leq \sum_{i, j \in I_{N}} \Lambda_{i j}^{2 \theta-1}(\Delta t)^{2 \theta}\left|\left\langle Q e_{i}, e_{j}\right\rangle\right| \cdot\left|\left(e_{i}(x)-e_{i}(y)\right)\left(e_{j}(x)-e_{j}(y)\right)\right| \\
& \leq C \sum_{i, j \in I_{N}} \Lambda_{i j}^{2 \theta-1}(\Delta t)^{2 \theta}\left|\left\langle Q e_{i}, e_{j}\right\rangle\right| \cdot\left(\|i\|_{2}^{2}\|x-y\|_{2}^{2}\right)^{\alpha}\left(\|j\|_{2}^{2}\|x-y\|_{2}^{2}\right)^{\alpha} \\
& \leq C(\Delta t)^{2 \theta}\|x-y\|_{2}^{4 \alpha} \sum_{i, j \in I_{N}}\left(\|i\|_{2}^{2}+\|j\|_{2}^{2}\right)^{2 \theta-1}\|i\|_{2}^{2 \alpha}\|j\|_{2}^{2 \alpha}\left|\left\langle Q e_{i}, e_{j}\right\rangle\right|,
\end{aligned}
$$

where we used that $e_{k}$ is bounded and Lipschitz. Therefore,

$$
\begin{aligned}
& \mathbb{E}\left|\left(O_{t_{2}}^{N}(x)-O_{t_{1}}^{N}(x)\right)-\left(O_{t_{2}}^{N}(y)-O_{t_{1}}^{N}(y)\right)\right|^{2} \\
& \leq C(\Delta t)^{2 \theta}\|x-y\|_{2}^{4 \alpha} \sum_{i, j \in I_{N}}\|i\|_{2}^{2 \theta+2 \alpha-1}\|j\|_{2}^{2 \theta+2 \alpha-1}\left|\left\langle Q e_{i}, e_{j}\right\rangle\right| .
\end{aligned}
$$

Again from Lemma 5 we derive in a similar way for every $x \in[0,1]^{d}$

$$
\begin{aligned}
\mathbb{E}\left[\left|O_{t_{2}}^{N}(x)-O_{t_{1}}^{N}(x)\right|^{2}\right] & \leq C \sum_{i, j \in I_{N}} \Lambda_{i j}^{2 \theta-1}(\Delta t)^{2 \theta}\left|\left\langle Q e_{i}, e_{j}\right\rangle\right| \\
& \leq C \sum_{i, j \in I_{N}}\left(\|i\|_{2}^{2}+\|j\|_{2}^{2}\right)^{2 \theta-1}(\Delta t)^{2 \theta}\left|\left\langle Q e_{i}, e_{j}\right\rangle\right| .
\end{aligned}
$$


The Sobolev embedding of the fractional space $W^{\alpha, p}$ into $C^{0}\left([0,1]^{d}\right)$ given in Theorem 1 in Section 2.2.4 in [20] yields

$$
\begin{aligned}
& \mathbb{E}\left[\left\|O_{t_{2}}^{N}-O_{t_{1}}^{N}\right\|_{C^{0}\left([0,1]^{d}\right)}^{p}\right] \\
& \leq C \int_{(0,1)^{d}} \int_{(0,1)^{d}} \frac{\left(\mathbb{E}\left[\left|\left(O_{t_{2}}^{N}(x)-O_{t_{1}}^{N}(x)\right)-\left(O_{t_{2}}^{N}(y)-O_{t_{1}}^{N}(y)\right)\right|^{2}\right]\right)^{\frac{p}{2}}}{\|x-y\|_{2}^{d+p \alpha}} d x d y \\
& +C \int_{(0,1)^{d}}\left(\mathbb{E}\left[\left|O_{t_{2}}^{N}(x)-O_{t_{1}}^{N}(x)\right|^{2}\right]\right)^{\frac{p}{2}} d x,
\end{aligned}
$$

where we have used Gaussianity for the $p$-th moment. In the following, for shorthand notation, all spatial integrals are over $(0,1)^{d}$.

Therefore, by (14) and (15)

$$
\begin{aligned}
& \mathbb{E}\left[\left\|O_{t_{2}}^{N}-O_{t_{1}}^{N}\right\|_{C^{0}\left([0,1]^{d}\right)}^{p}\right] \\
& \leq C \iint \frac{\left((\Delta t)^{2 \theta}\|x-y\|_{2}^{4 \alpha}\right)^{\frac{p}{2}}}{\|x-y\|_{2}^{d+p \alpha}} d x d y\left(\sum_{i, j \in I_{N}}\left(\|i\|_{2}\|j\|_{2}\right)^{2 \theta+2 \alpha-1}\left|\left\langle Q e_{i}, e_{j}\right\rangle\right|\right)^{\frac{p}{2}} \\
& \quad+C \int(\Delta t)^{p \theta}\left(\sum_{i, j \in I_{N}}\|i\|_{2}^{2 \theta-1}\|j\|_{2}^{2 \theta-1}\left|\left\langle Q e_{i}, e_{j}\right\rangle\right|\right)^{\frac{p}{2}} d x \\
& \leq C\left(1+\iint\|x-y\|_{2}^{\alpha p-d} d x d y\right) \cdot(\Delta t)^{p \theta} \cdot\left(\sum_{i, j \in I_{N}}\left(\|i\|_{2}\|j\|_{2}\right)^{2 \theta+2 \alpha-1}\left|\left\langle Q e_{i}, e_{j}\right\rangle\right|\right)^{\frac{p}{2}} .
\end{aligned}
$$

By the fact that with arbitrary $d \in \mathbb{N}$

$$
\iint\left(\|x-y\|_{2}\right)^{-\alpha} d x d y \leq \frac{(3 d)^{d}}{d-\alpha}
$$

for every $\alpha \in(0, d)$, we derive

$$
\left(\mathbb{E}\left\|O_{t_{2}}^{N}-O_{t_{1}}^{N}\right\|_{C^{0}\left([0,1]^{d}\right)}^{p}\right)^{\frac{1}{p}} \leq C\left(\sum_{i, j \in I_{N}}\left(\|i\|_{2}\|j\|_{2}\right)^{2 \theta+2 \alpha-1}\left|\left\langle Q e_{i}, e_{j}\right\rangle\right|\right)^{\frac{1}{2}}(\Delta t)^{\theta} .
$$

Lemma 7. For every $N, M \in \mathbb{N}, N \geq M, p \in[1, \infty)$ and every $\alpha \in\left(0, \frac{1}{2}\right]$ we have

$$
\left(\mathbb{E} \sup _{0 \leq t \leq T}\left\|O_{t}^{N}-O_{t}^{M}\right\|_{C^{0}\left([0,1]^{d}\right)}^{p}\right)^{\frac{1}{p}} \leq C\left(\sum_{i, j \in I_{N} \backslash I_{M}}\|i\|_{2}^{4 \alpha-1}\|j\|_{2}^{4 \alpha-1}\left|\left\langle Q e_{i}, e_{j}\right\rangle\right|\right)^{\frac{1}{2}}
$$

where $I_{N}=\{1, \ldots, N\}^{d}, I_{M}=\{1, \ldots, M\}^{d}$ and $C=C(d, p, \alpha, \theta)$ is a constant only depending on $d, p, \alpha$, and $\theta$.

Proof. Throughout this proof we assume $\alpha \in\left(0, \frac{1}{2}\right)$ and $p>\frac{1}{\alpha}$. Moreover, $N>M$ is fixed. Define for every $t \in[0, T]$

$$
Y_{t}^{N, M}=\sum_{i \in I_{N} \backslash I_{M}} \int_{0}^{t}(t-s)^{-\alpha} e^{-\lambda_{i}(t-s)} d \beta_{s}^{i} e_{i}
$$


The celebrated factorization method [6] yields

$$
\begin{aligned}
& \mathbb{E} \sup _{0 \leq t \leq T}\left\|O_{t}^{N}-O_{t}^{M}\right\|_{C^{0}\left([0,1]^{d}\right)}^{p} \\
& =\mathbb{E} \sup _{0 \leq t \leq T}\left\|\frac{\sin (\pi \alpha)}{\pi} \int_{0}^{t}(t-s)^{\alpha-1} S_{t-s} Y_{s}^{N, M} d s\right\|_{C^{0}\left([0,1]^{d}\right)}^{p} \\
& \leq \mathbb{E} \sup _{0 \leq t \leq T}\left\|\int_{0}^{t}(t-s)^{\alpha-1} S_{t-s} Y_{s}^{N, M} d s\right\|_{C^{0}\left([0,1]^{d}\right)}^{p}
\end{aligned}
$$

Therefore, using Hölder inequality and boundedness of $\left\|S_{t}\right\|_{L\left(C^{0}\left([0,1]^{d}\right)\right)}$ yields

$$
\begin{aligned}
& \mathbb{E} \sup _{0 \leq t \leq T}\left\|O_{t}^{N}-O_{t}^{M}\right\|_{C^{0}\left([0,1]^{d}\right)}^{p} \\
& \leq \sup _{0 \leq t \leq T}\left(\int_{0}^{t}(t-s)^{\frac{p(\alpha-1)}{p-1}} d s\right)^{p-1} \cdot \mathbb{E} \int_{0}^{T}\left\|Y_{s}^{N, M}\right\|_{C^{0}\left([0,1]^{d}\right)}^{p} d s \\
& \leq C \int_{0}^{T} \mathbb{E}\left\|Y_{s}^{N, M}\right\|_{C^{0}\left([0,1]^{d}\right)}^{p} d s .
\end{aligned}
$$

Hence,

$$
\left(\mathbb{E} \sup _{0 \leq t \leq T}\left\|O_{t}^{N}-O_{t}^{M}\right\|_{C^{0}\left([0,1]^{d}\right)}^{p}\right)^{\frac{1}{p}} \leq C \sup _{0 \leq t \leq T}\left(\mathbb{E}\left\|Y_{t}^{N, M}\right\|_{C^{0}\left([0,1]^{d}\right)}^{p}\right)^{\frac{1}{p}} .
$$

Again using the embedding of $W^{\alpha, p}$ into $C^{0}$

$$
\begin{aligned}
\mathbb{E}\left\|Y_{t}^{N, M}\right\|_{C^{0}\left([0,1]^{d}\right)}^{p} & \leq C \int_{(0,1)^{d}} \int_{(0,1)^{d}} \frac{\left(\mathbb{E}\left|Y_{t}^{N, M}(x)-Y_{t}^{N, M}(y)\right|^{2}\right)^{\frac{p}{2}}}{\|x-y\|_{2}^{d+p \alpha}} d x d y \\
& +C \int_{(0,1)^{d}}\left(\mathbb{E}\left|Y_{t}^{N, M}(x)\right|^{2}\right)^{\frac{p}{2}} d x .
\end{aligned}
$$

For the first term on the right side of (17) we proceed completely analogous to Lemma 6, in order to obtain

$$
\begin{aligned}
& \mathbb{E}\left|Y_{t}^{N, M}(x)-Y_{t}^{N, M}(y)\right|^{2} \\
& \leq C \sum_{i, j \in I_{N} \backslash I_{M}} \int_{0}^{\infty} s^{-2 \alpha} e^{-s} d s \cdot\left(\lambda_{i}+\lambda_{j}\right)^{2 \alpha-1} \cdot\left|\left\langle Q e_{i}, e_{j}\right\rangle\right| \cdot\|i\|_{2}^{2 \alpha}\|j\|_{2}^{2 \alpha}\|x-y\|_{2}^{4 \alpha} .
\end{aligned}
$$

Therefore,

$$
\mathbb{E}\left|Y_{t}^{N, M}(x)-Y_{t}^{N, M}(y)\right|^{2} \leq C \sum_{i, j \in I_{N} \backslash I_{M}} \frac{\left|\left\langle Q e_{i}, e_{j}\right\rangle\right|}{\|i\|_{2}^{1-4 \alpha}\|j\|_{2}^{1-4 \alpha}}\|x-y\|_{2}^{4 \alpha} .
$$

For the second term on the right hand side of (17) we establish

$$
\begin{aligned}
\mathbb{E}\left|Y_{t}^{N, M}(x)\right|^{2} & \leq \sum_{i, j \in I_{N} \backslash I_{M}} \int_{0}^{t}(t-s)^{-2 \alpha} e^{-\left(\lambda_{i}+\lambda_{j}\right)(t-s)} d s\left|\left\langle Q e_{i}, e_{j}\right\rangle\left\|e_{i}(x)\right\| e_{j}(x)\right| \\
& \leq C \sum_{i, j \in I_{N} \backslash I_{M}}\|i\|_{2}^{2 \alpha-1}\|j\|_{2}^{2 \alpha-1}\left|\left\langle Q e_{i}, e_{j}\right\rangle\right| .
\end{aligned}
$$


Hence using (18) and (19) we obtain from (17)

$$
\sup _{0 \leq t \leq T}\left(\mathbb{E}\left\|Y_{t}^{N, M}\right\|_{C^{0}\left([0,1]^{d}\right)}^{p}\right)^{\frac{1}{p}} \leq C\left(\sum_{i, j \in I_{N} \backslash I_{M}}\|i\|_{2}^{4 \alpha-1}\|j\|_{2}^{4 \alpha-1}\left|\left\langle Q e_{i}, e_{j}\right\rangle\right|\right)^{\frac{1}{2}} .
$$

Finally, (16) and (20) yield

$$
\left(\mathbb{E} \sup _{0 \leq t \leq T}\left\|O_{t}^{N}(x)-O_{t}^{M}(x)\right\|_{C^{0}\left([0,1]^{d}\right)}^{p}\right)^{\frac{1}{p}} \leq C\left(\sum_{i, j \in I_{N} \backslash I_{M}}\|i\|_{2}^{4 \alpha-1}\|j\|_{2}^{4 \alpha-1}\left\langle Q e_{i}, e_{j}\right\rangle\right)^{\frac{1}{2}} .
$$

Now we are ready to present the remaining parts of the proof of Lemma 4 .

Proof. (Proof of Lemma 4). From Lemma 7 we obtain

$$
\begin{aligned}
\left(\mathbb{E}\left[\sup _{0 \leq t \leq T}\left\|O_{t}^{N}-O_{t}^{M}\right\|_{C^{0}\left([0,1]^{d}\right)}^{p}\right]\right)^{\frac{1}{p}} & \leq C\left(\sum_{i, j \in \mathbb{N}^{d} \backslash I_{M}}\|i\|_{2}^{4 \alpha-1}\|j\|_{2}^{4 \alpha-1}\left|\left\langle Q e_{i}, e_{j}\right\rangle\right|\right)^{\frac{1}{2}} \\
& \leq C M^{4 \alpha-\rho}\left(\sum_{i, j \in \mathbb{N}^{d}}\|i\|_{2}^{\rho-1}\|j\|_{2}^{\rho-1}\left|\left\langle Q e_{i}, e_{j}\right\rangle\right|\right)^{\frac{1}{2}}
\end{aligned}
$$

for every $N, M \in \mathbb{N}$ with $N \geq M, p \in[1, \infty)$, and $\alpha \in\left(0, \min \left\{\frac{1}{2}, \frac{\rho}{4}\right\}\right)$. The processes $O^{N}$ form a Cauchy sequences in

$$
V_{p}:=L^{p}\left((\Omega, \mathcal{F}, \mathbb{P}),\left(C^{0}\left([0, T] \times[0,1]^{d}\right)\right)\right) .
$$

Hence, there exists a stochastic process $\tilde{O}:[0, T] \times \Omega \rightarrow C^{0}\left([0,1]^{d}\right)$ with $\tilde{O} \in V_{p}$ and

$$
\left(\mathbb{E} \sup _{0 \leq t \leq T}\left\|\tilde{O}_{t}-O_{t}^{N}\right\|_{C^{0}\left([0,1]^{d}\right)}^{p}\right)^{\frac{1}{p}} \leq C N^{4 \alpha-\rho}\left(\sum_{i, j \in \mathbb{N}^{d}}\|i\|_{2}^{\rho-1}\|j\|_{2}^{\rho-1} \mid\left\langle Q e_{i}, e_{j}\right\rangle\right)^{\frac{1}{2}}
$$

for every $N \in \mathbb{N}, p \in[1, \infty)$, and $\alpha \in\left(0, \min \left\{\frac{1}{2}, \frac{\rho}{4}\right\}\right)$.

Therefore,

$$
\sup _{N \in \mathbb{N}}\left\{N^{\gamma}\left(\mathbb{E} \sup _{0 \leq t \leq T}\left\|\tilde{O}_{t}-O_{t}^{N}\right\|_{C^{0}\left([0,1]^{d}\right)}^{p}\right)^{\frac{1}{p}}\right\}<\infty
$$

for every $\gamma \in(0, \rho)$ and every $p \in[1, \infty)$. This yields (Lemma 1 in [14])

$$
\mathbb{P}\left[\sup _{N \in \mathbb{N}} \sup _{0 \leq t \leq T}\left\{N^{\gamma}\left\|\tilde{O}_{t}-O_{t}^{N}\right\|_{C^{0}\left([0,1]^{d}\right)}\right\}<\infty\right]=1 .
$$

In particular,

$$
\mathbb{P}\left[\lim _{N \rightarrow \infty} \sup _{0 \leq t \leq T}\left\|\tilde{O}_{t}-O_{t}^{N}\right\|_{C^{0}\left([0,1]^{d}\right)}=0\right]=1
$$

and

$$
\mathbb{P}\left[\sup _{N \in \mathbb{N} 0 \leq t \leq T} \sup _{0 \leq t}\left\{N^{\gamma}\left\|\tilde{O}_{t}-P_{N} \tilde{O}_{t}\right\|_{C^{0}\left([0,1]^{d}\right)}\right\}<\infty\right]=1 .
$$


From Lemma 6 we derive

$$
\begin{aligned}
\left(\mathbb{E} \| O_{t_{2}}^{N}-\right. & \left.O_{t_{1}}^{N} \|_{C^{0}\left([0,1]^{d}\right)}^{p}\right)^{\frac{1}{p}} \\
\leq & C\left(\sum_{i, j \in I_{N}}\left(\|i\|_{2}\|j\|_{2}\right)^{2 \theta+2\left(\frac{\rho}{2}-\theta\right)-1}\left|\left\langle Q e_{i}, e_{j}\right\rangle\right|\right)^{\frac{1}{2}}\left|t_{2}-t_{1}\right|^{\theta} \\
\leq & C\left(\sum_{i, j \in I_{N}}\|i\|_{2}^{\rho-1}\|j\|_{2}^{\rho-1}\left|\left\langle Q e_{i}, e_{j}\right\rangle\right|\right)^{\frac{1}{2}}\left|t_{2}-t_{1}\right|^{\theta}
\end{aligned}
$$

for every $t_{1}, t_{2} \in[0, T], N \in \mathbb{N}$, and $\theta \in\left(0, \frac{\rho}{2}\right)$. Provided $\theta \leq \frac{1}{2}$ this furnishes

$$
\left(\mathbb{E}\left\|\tilde{O}_{t_{2}}-\tilde{O}_{t_{1}}\right\|_{C^{0}\left([0,1]^{d}\right)}^{p}\right)^{\frac{1}{p}} \leq C\left(\sum_{i, j \in I_{N}}\|i\|_{2}^{\rho-1}\|j\|_{2}^{\rho-1}\left|\left\langle Q e_{i}, e_{j}\right\rangle\right|\right)^{\frac{1}{2}}\left|t_{2}-t_{1}\right|^{\theta} .
$$

Hence, for every $\theta \in\left(0, \min \left\{\frac{1}{2}, \frac{\rho}{2}\right\}\right)$

$$
\mathbb{P}\left[\sup _{0 \leq t_{1}, t_{2} \leq T} \frac{\left\|\tilde{O}_{t_{2}}-\tilde{O}_{t_{1}}\right\|_{C^{0}\left([0,1]^{d}\right)}}{\left|t_{2}-t_{1}\right|^{\theta}}<\infty\right]=1 .
$$

Therefore,

$$
\mathbb{P}\left[\forall \theta \in\left(0, \min \left\{\frac{1}{2}, \frac{\rho}{2}\right\}\right): \sup _{0 \leq t_{1}, t_{2} \leq T} \frac{\left\|\tilde{O}_{t_{2}}-\tilde{O}_{t_{1}}\right\|_{C^{0}\left([0,1]^{d}\right)}}{\left|t_{2}-t_{1}\right|^{\theta}}<\infty\right]=1 .
$$

In conclusion, this shows the existence of a process $O:[0, T] \times \Omega \rightarrow C^{0}\left([0,1]^{d}\right)$, which satisfies

$$
\sup _{0 \leq t_{1}, t_{2} \leq T} \frac{\left\|O_{t_{2}}(\omega)-O_{t_{1}}(\omega)\right\|_{C^{0}\left([0,1]^{d}\right)}}{\left|t_{2}-t_{1}\right|^{\theta}}<\infty
$$

and

$$
\sup _{N \in \mathbb{N}} \sup _{0 \leq t \leq T}\left(N^{\gamma}\left\|O_{t}(\omega)-P_{N} O_{t}(\omega)\right\|_{C^{0}\left([0,1]^{d}\right)}\right)<\infty
$$

for every $\omega \in \Omega, \theta \in\left(0, \min \left\{\frac{1}{2}, \frac{\rho}{2}\right\}\right)$, and $\gamma \in(0, \rho)$. Moreover, $O$ is indistinguishable from $\tilde{O}$, i.e.,

$$
\mathbb{P}\left[\forall t \in[0, T]: O_{t}=\tilde{O}_{t}\right]=1 .
$$

Summarizing our results, we can state the following Lemma:

Lemma 8. Assume $\rho>0, d \in\{1,2,3\}$ and

$$
\sum_{i, j \in N^{d}}\|i\|_{2}^{\rho-1}\|j\|_{2}^{\rho-1}\left|\left\langle Q e_{i}, e_{j}\right\rangle\right|<\infty .
$$

Furthermore, suppose that $\xi: \Omega \rightarrow V$ is $\mathcal{F} / V$-measurable with

$$
\sup _{N \in \mathbb{N}}\left(N^{\rho}\left\|\xi(\omega)-P_{N}(\xi(\omega))\right\|_{V}\right)<\infty
$$


for every $\omega \in \Omega$. Then there exists a stochastic process $O:[0, T] \times \Omega \rightarrow V$ with continuous sample paths, satisfying

$$
\mathbb{P}\left[\lim _{N \rightarrow \infty} \sup _{0<t<T}\left\|O_{t}-S_{t} \xi-\sum_{i \in I_{N}}\left(-\lambda_{i} \int_{0}^{t} e^{-\lambda_{i}(t-s)} \beta_{s}^{i} d s+\beta_{t}^{i}\right) e_{i}\right\|_{V}=0\right]=1
$$

and

$$
\sup _{N \in \mathbb{N}} \sup _{0 \leq t \leq T}\left\{N^{\gamma}\left\|O_{t}(\omega)-P_{N}\left(O_{t}(\omega)\right)\right\|_{V}\right\}<\infty
$$

for every $\omega \in \Omega$ and $\gamma \in(0, \rho)$.

In particular $O$ satisfies Assumptions 3 for every $\gamma \in(0, \rho)$.

Note that the process $O$ in the previous Lemma 8 is the solution of the following linear SPDE

$$
d O_{t}=\Delta O_{t} d t+d W_{t},\left.\quad O_{t}\right|_{\partial(0,1)^{d}}=0, \quad O_{0}=\xi,
$$

for $t \in[0, T]$, where $W$ is a $Q$-Wiener process.

Lemma 9. Let $V=C^{0}([0,1]), W=H^{-1}((0,1))$ and $S:(0, T] \rightarrow L(W, V)$, and $F: V \rightarrow W$ be given by Lemmas 2, 3. Let $O:[0, T] \times \Omega \rightarrow V$ be a stochastic process with continuous sample paths with

$$
\sup _{N \in \mathbb{N}} \sup _{0 \leq t \leq T}\left\|P_{N}\left(O_{t}(\omega)\right)\right\|_{V}<\infty
$$

for every $\omega \in \Omega$. Then Assumption 4 is fulfilled.

Proof. The proof is exactly the same as the one of Lemma 4.9 in [3].

\section{Time discretization}

For time discretization of the finite dimensional SDEs (6) we study the method introduced by Jentzen, Kloeden and Winkel in [15]. Consider the discretization scheme for the Burgers equation, i.e., $F(u)=\partial_{x} u^{2}$ in one dimension. This is for simplicity of presentation only, as we need to bound various terms depending on $X^{N}$ and $F\left(X^{N}\right)$.

Through this section assume $\rho>0$, is such that

$$
\sum_{i, j \in N^{d}}\|i\|_{2}^{\rho-1}\|j\|_{2}^{\rho-1}\left|\left\langle Q e_{i}, e_{j}\right\rangle\right|<\infty .
$$

Moreover assume $\theta \in\left(0, \min \left\{\frac{1}{2}, \frac{\rho}{2}\right\}\right)$. For the time discretization we define the mapping $Y_{m}^{N, M}: \Omega \rightarrow V$ for $m \in\{1, \ldots, M\}$ by

$Y_{m+1}^{N, M}(\omega)=S_{\Delta t}\left(Y_{m}^{N, M}(\omega)+\Delta t\left(P_{N} F\right)\left(Y_{m}^{N, M}(\omega)\right)\right)+P_{N}\left(O_{(m+1) \Delta t}(\omega)-S_{\Delta t} O_{m \Delta t}(\omega)\right)$.

The purpose of this section is to consider the discretization error in time

$$
\left\|X_{m \Delta t}^{N}(\omega)-Y_{m}^{N, M}(\omega)\right\|_{V}
$$


where

$$
X_{m \Delta t}^{N}(\omega)=\int_{0}^{m \Delta t} P_{N} S_{m \Delta t-s} F\left(X_{s}^{N}(\omega)\right) d s+O_{m \Delta t}^{N}(\omega),
$$

is the solution of the spatial discretization, which is evaluated at the grid points.

Recall that as we proved in the last section Assumptions 1-4 are satisfied for the stochastic Burgers equation in one dimension.

Lemma 10. Let $X^{N}:[0, T] \times \Omega \rightarrow V$ be the unique adapted stochastic process with continuous sample paths, defined in Assumption 4. Assume that $O^{N}$ : $[0, T] \times \Omega \rightarrow C^{0}\left([0,1]^{d}\right)$ is the stochastic process defined in (13). Then we obtain

$$
\left\|\left(X_{t_{2}}^{N}(\omega)-O_{t_{2}}^{N}(\omega)\right)-\left(X_{t_{1}}^{N}(\omega)-O_{t_{1}}^{N}(\omega)\right)\right\|_{V} \leq C(\omega)\left(t_{2}-t_{1}\right)^{\frac{1}{4}}
$$

for every $\omega \in \Omega$ and all $t_{1}, t_{2} \in[0, T]$, with $t_{1}<t_{2}$ where $C$ is a finite random variable $C: \Omega \rightarrow[0, \infty)$.

Proof. For every $0 \leq t_{1} \leq t_{2} \leq T$ we have

$$
\begin{aligned}
& \left\|X_{t_{2}}^{N}(\omega)-O_{t_{2}}^{N}(\omega)-\left(X_{t_{1}}^{N}(\omega)-O_{t_{1}}^{N}(\omega)\right)\right\|_{V} \\
& =\left\|\int_{0}^{t_{2}} P_{N} S_{t_{2}-s} F\left(X_{s}^{N}(\omega)\right) d s-\int_{0}^{t_{1}} P_{N} S_{t_{1}-s} F\left(X_{s}^{N}(\omega)\right) d s\right\|_{V} \\
& =\left\|\int_{t_{1}}^{t_{2}} P_{N} S_{t_{2}-s} F\left(X_{s}^{N}(\omega)\right) d s+\int_{0}^{t_{1}}\left(S_{t_{2}-s}-S_{t_{1}-s}\right) P_{N} F\left(X_{s}^{N}(\omega)\right) d s\right\|_{V} \\
& \leq \int_{t_{1}}^{t_{2}}\left\|P_{N} S_{t_{2}-s} \partial\right\|_{L(V, V)}\left\|\left(X_{s}^{N}(\omega)\right)^{2}\right\|_{V} d s+\left\|\int_{0}^{t_{1}} S_{t_{1}-s}\left(S_{t_{2}-t_{1}}-I\right) P_{N} F\left(X_{s}^{N}(\omega)\right) d s\right\|_{V} .
\end{aligned}
$$

From (9) and using the fact that $S_{t}$ is the semigroup generated by Laplacian operator, $\Delta$, we conclude

$$
\begin{aligned}
& \left\|X_{t_{2}}^{N}(\omega)-O_{t_{2}}^{N}(\omega)-\left(X_{t_{1}}^{N}(\omega)-O_{t_{1}}^{N}(\omega)\right)\right\|_{V} \\
& \leq C_{1}(\omega) \int_{t_{1}}^{t_{2}}\left(t_{2}-s\right)^{-\frac{3}{4}} d s+\int_{0}^{t_{1}}\left\|P_{N} S_{t_{1}-s} \Delta^{\frac{1}{4}}\right\|_{L(W, V)}\left\|\left(S_{t_{2}-t_{1}}-I\right) \Delta^{-\frac{1}{4}}\right\|_{L(W, V)}\left\|F\left(X_{s}^{N}(\omega)\right)\right\|_{W} d s \\
& \leq 4 C_{1}(\omega)\left(t_{2}-t_{1}\right)^{\frac{1}{4}}+\int_{0}^{t_{1}}\left(t_{1}-s\right)^{-\frac{1}{4}} d s\left(t_{2}-t_{1}\right)^{\frac{1}{4}}\left\|F\left(X_{s}^{N}(\omega)\right)\right\|_{W} \\
& \leq 4 C_{1}(\omega)\left(t_{2}-t_{1}\right)^{\frac{1}{4}}+C_{2}(\omega)\left(t_{2}-t_{1}\right)^{\frac{1}{4}} T^{\frac{3}{4}} \\
& \leq C(\omega)\left(t_{2}-t_{1}\right)^{\frac{1}{4}} .
\end{aligned}
$$

where $C_{1}(\omega)=\sup _{M \in \mathbb{N}} \sup _{0 \leq s \leq T}\left\|X_{s}^{M}(\omega)\right\|_{V}^{2}, C_{2}(\omega)=\sup _{M \in \mathbb{N}} \sup _{0 \leq s \leq T}\left\|F\left(X_{s}^{M}(\omega)\right)\right\|_{W}$ are finite due to Assumptions 4 and 2 , and therefore $C$ is an almost surely finite random variable $C: \Omega \rightarrow[0, \infty)$.

Before we begin with the first part of the error, we define

$$
\begin{aligned}
R(\omega):= & \sup _{N \in \mathbb{N}} \sup _{0 \leq s \leq T}\left\|F\left(X_{s}^{N}(\omega)\right)\right\|_{W}+\sup _{N \in \mathbb{N}} \sup _{0 \leq s \leq T}\left\|X_{s}^{N}(\omega)\right\|_{V} \\
& +\sup _{0 \leq t_{1}, t_{2} \leq T}\left\|O_{t_{2}}(\omega)-O_{t_{1}}(\omega)\right\|_{V}\left|t_{2}-t_{1}\right|^{-\theta} \\
& +\sup _{N \in \mathbb{N}} \sup _{0 \leq t_{1}, t_{2} \leq T}\left\|X_{t_{2}}^{N}(\omega)-O_{t_{2}}^{N}(\omega)-\left(X_{t_{1}}^{N}(\omega)-O_{t_{1}}^{N}(\omega)\right)\right\|_{V}\left|t_{2}-t_{1}\right|^{-\frac{1}{4}},
\end{aligned}
$$


where from Assumption 4, Lemma 4 and Lemma 10, $R: \Omega \rightarrow \mathbb{R}$ is a finite random variable.

The main result of this section is stated below.

Theorem 11. For $m \in\{0,1, \ldots, M\}$ and every $M, N \in \mathbb{N}$, there exists a finite random variable $C: \Omega \rightarrow[0, \infty)$ such that

$$
\left\|X_{m \Delta t}^{N}(\omega)-Y_{m}^{N, M}(\omega)\right\|_{V} \leq C(\omega)(\Delta t)^{\min \left(\frac{1}{4}, \theta\right)},
$$

where $X^{N}:[0, T] \times \Omega \rightarrow V$ is the unique adapted stochastic process with continuous sample paths, defined in Assumption 4, and $Y_{m}^{N, M}: \Omega \rightarrow V$, for $m \in\{0,1, \ldots, M\}$, and $N, M \in \mathbb{N}$, is given in (22).

Proof. For the proof it is sufficient to prove the result for sufficiently small $\left|t_{2}-t_{1}\right|$. Due to (6) we have

$$
\begin{aligned}
X_{m \Delta t}^{N}(\omega) & =\int_{0}^{m \Delta t} P_{N} S_{m \Delta t-s} F\left(X_{s}^{N}(\omega)\right) d s+O_{m \Delta t}^{N}(\omega) \\
& =\sum_{k=0}^{m-1} \int_{k \Delta t}^{(k+1) \Delta t} P_{N} S_{m \Delta t-s} F\left(X_{s}^{N}(\omega)\right) d s+O_{m \Delta t}^{N}(\omega),
\end{aligned}
$$

for every $m \in\{0,1, \ldots, M\}$, and every $M \in \mathbb{N}$.

The mapping $Y_{m}^{N}: \Omega \rightarrow V, m=1,2, \ldots, M$ is defined by

$$
Y_{m}^{N}(\omega)=\sum_{k=0}^{m-1} \int_{k \Delta t}^{(k+1) \Delta t} P_{N} S_{m \Delta t-k \Delta t} F\left(X_{k \Delta t}^{N}(\omega)\right) d s+O_{m \Delta t}^{N}(\omega) .
$$

Our aim is to bound $\left\|X_{m \Delta t}^{N}(\omega)-Y_{m}^{N, M}(\omega)\right\|_{V}$. Therefore, we first estimate the difference of the true solution to $Y_{m}^{N}$

$$
\left\|X_{m \Delta t}^{N}(\omega)-Y_{m}^{N}(\omega)\right\|_{V}
$$

for every $m \in\{0,1, \ldots, M\}$ and then the difference between $Y_{m}^{N}$ and the full discretization in time

$$
\left\|Y_{m}^{N}(\omega)-Y_{m}^{N, M}(\omega)\right\|_{V}
$$

For the first error in (25) we have

$$
\begin{aligned}
& X_{m \Delta t}^{N}(\omega)-Y_{m}^{N}(\omega) \\
= & \sum_{k=0}^{m-2} \int_{k \Delta t}^{(k+1) \Delta t} P_{N} S_{m \Delta t-s} F\left(X_{s}^{N}(\omega)\right) d s \\
& -\sum_{k=0}^{m-2} \int_{k \Delta t}^{(k+1) \Delta t} P_{N} S_{m \Delta t-k \Delta t} F\left(X_{k \Delta t}^{N}(\omega)\right) d s \\
& +\int_{(m-1) \Delta t}^{m \Delta t} P_{N} S_{m \Delta t-s} F\left(X_{s}^{N}(\omega)\right) d s-\int_{(m-1) \Delta t}^{m \Delta t} P_{N} S_{\Delta t} F\left(X_{k \Delta t}^{N}(\omega)\right) d s .
\end{aligned}
$$


Let us now bound the last two integrals in (27). For the first one, we derive

$$
\begin{aligned}
\| \int_{(m-1) \Delta t}^{m \Delta t} P_{N} & S_{m \Delta t-s} F\left(X_{s}^{N}(\omega)\right) d s \|_{V} \\
& =\left\|\int_{(m-1) \Delta t}^{m \Delta t} P_{N} S_{m \Delta t-s} \partial\left(X_{s}^{N}(\omega)\right)^{2} d s\right\|_{V} \\
& \leq \int_{(m-1) \Delta t}^{m \Delta t}\left\|P_{N} S_{m \Delta t-s} \partial\right\|_{L(V, V)} \cdot\left\|X_{s}^{N}(\omega)\right\|_{V}^{2} d s \\
& \leq \sup _{0 \leq s \leq t}\left\|X_{s}^{N}(\omega)\right\|_{V}^{2} \int_{(m-1) \Delta t}^{m \Delta t}(m \Delta t-s)^{-\frac{3}{4}} d s \\
& \leq R^{2}(\omega)(\Delta t)^{\frac{1}{4}} .
\end{aligned}
$$

For the second one we get

$$
\begin{aligned}
\left\|\int_{(m-1) \Delta t}^{m \Delta t} P_{N} S_{\Delta t} F\left(X_{k \Delta t}^{N}(\omega)\right) d s\right\|_{V} & =\left\|\int_{(m-1) \Delta t}^{m \Delta t} P_{N} S_{\Delta t} \partial\left(X_{k \Delta t}^{N}(\omega)\right)^{2} d s\right\|_{V} \\
& \leq \int_{(m-1) \Delta t}^{m \Delta t}\left\|P_{N} S_{\Delta t} \partial\right\|_{L(V, V)} \cdot\left\|X_{k \Delta t}^{N}(\omega)\right\|_{V}^{2} d s \\
& \leq \sup _{0 \leq s \leq t}\left\|X_{s}^{N}(\omega)\right\|_{V}^{2} \int_{(m-1) \Delta t}^{m \Delta t}(\Delta t)^{-\frac{3}{4}} d s \\
& \leq R^{2}(\omega)(\Delta t)^{\frac{1}{4}} .
\end{aligned}
$$

Therefore, we can conclude

$$
\begin{aligned}
& \left\|X_{m \Delta t}^{N}(\omega)-Y_{m}^{N}(\omega)\right\|_{V} \\
& \leq\left\|\sum_{k=0}^{m-2} \int_{k \Delta t}^{(k+1) \Delta t} P_{N} S_{m \Delta t-s}\left(F\left(X_{s}^{N}(\omega)\right)-F\left(X_{k \Delta t}^{N}(\omega)\right)\right) d s\right\|_{V} \\
& +\left\|\sum_{k=0}^{m-2} \int_{k \Delta t}^{(k+1) \Delta t}\left(P_{N} S_{m \Delta t-s}-P_{N} S_{(m \Delta t-k \Delta t)}\right) F\left(X_{k \Delta t}^{N}(\omega)\right) d s\right\|_{V} \\
& +R^{2}(\omega)(\Delta t)^{\frac{1}{4}} .
\end{aligned}
$$

Thus inserting the nonlinearity with the Ornstein-Uhlenbeck process in the first term yields for every $m \in\{0,1, \ldots, M\}$,

$$
\begin{aligned}
& \left\|X_{m \Delta t}^{N}(\omega)-Y_{m}^{N}(\omega)\right\|_{V} \\
& \leq\left\|\sum_{k=0}^{m-2} \int_{k \Delta t}^{(k+1) \Delta t} P_{N} S_{m \Delta t-s}\left(F\left(X_{s}^{N}(\omega)\right)-F\left(X_{k \Delta t}^{N}(\omega)+O_{s}^{N}(\omega)-O_{k \Delta t}^{N}(\omega)\right)\right) d s\right\|_{V} \\
& +\left\|\sum_{k=0}^{m-2} \int_{k \Delta t}^{(k+1) \Delta t} P_{N} S_{m \Delta t-s}\left(F\left(X_{k \Delta t}^{N}(\omega)+O_{s}^{N}(\omega)-O_{k \Delta t}^{N}(\omega)\right)-F\left(X_{k \Delta t}^{N}(\omega)\right)\right) d s\right\|_{V} \\
& +\left\|\sum_{k=0}^{m-2} \int_{k \Delta t}^{(k+1) \Delta t}\left(P_{N} S_{m \Delta t-s}-P_{N} S_{m \Delta t-k \Delta t}\right) F\left(X_{k \Delta t}^{N}(\omega)\right) d s\right\|_{V} \\
& +R^{2}(\omega)(\Delta t)^{\frac{1}{4}} .
\end{aligned}
$$


For the first term in (28) by using Lemma 10 together with $\left\|P_{N} S_{t-s} \partial u\right\|_{V} \leq$ $C(t-s)^{-\frac{3}{4}}\|u\|_{V}$ we conclude

$$
\begin{aligned}
& \left\|\sum_{k=0}^{m-2} \int_{k \Delta t}^{(k+1) \Delta t} P_{N} S_{m \Delta t-s}\left(F\left(X_{s}^{N}(\omega)\right)-F\left(X_{k \Delta t}^{N}(\omega)+O_{s}^{N}(\omega)-O_{k \Delta t}^{N}(\omega)\right)\right) d s\right\|_{V} \\
& \leq \sum_{k=0}^{m-2} \int_{k \Delta t}^{(k+1) \Delta t}(m \Delta t-s)^{-\frac{3}{4}}\left\|X_{s}^{N}(\omega)-\left(X_{k \Delta t}^{N}(\omega)+O_{s}^{N}(\omega)-O_{k \Delta t}^{N}(\omega)\right)\right\|_{V} \\
& \cdot\left\|X_{s}^{N}(\omega)+\left(X_{k \Delta t}^{N}(\omega)+O_{s}^{N}(\omega)-O_{k \Delta t}^{N}(\omega)\right)\right\|_{V} d s \\
& \leq R(\omega) \sum_{k=0}^{m-2} \int_{k \Delta t}^{(k+1) \Delta t}(m \Delta t-s)^{-\frac{3}{4}}(s-k \Delta t)^{\frac{1}{4}}\left(2 R(\omega)+R(\omega)(s-k \Delta t)^{\theta}\right) d s \\
& \leq 2 C(R(\omega), T)(\Delta t)^{\frac{1}{4}}
\end{aligned}
$$

where the constant depends on $R$ and $T$.

For the second term in (28) we derive

$$
\begin{aligned}
& \left\|\sum_{k=0}^{m-2} \int_{k \Delta t}^{(k+1) \Delta t} P_{N} S_{m \Delta t-s}\left(F\left(X_{k \Delta t}^{N}(\omega)+O_{s}^{N}(\omega)-O_{k \Delta t}^{N}(\omega)\right)-F\left(X_{k \Delta t}^{N}(\omega)\right)\right) d s\right\|_{V} \\
& \leq 2 \sum_{k=0}^{m-2} \int_{k \Delta t}^{(k+1) \Delta t}\left\|P_{N} S_{m \Delta t-s} \partial\left(X_{k \Delta t}^{N}(\omega) \cdot\left(O_{s}^{N}(\omega)-O_{k \Delta t}^{N}(\omega)\right)\right)\right\|_{V} d s \\
& +\sum_{k=0}^{m-2} \int_{k \Delta t}^{(k+1) \Delta t}\left\|P_{N} S_{m \Delta t-s} \partial\left(\left(O_{s}^{N}(\omega)-O_{k \Delta t}^{N}(\omega)\right)^{2}\right)\right\|_{V} d s \\
& \leq 2 \sum_{k=0}^{m-2} \int_{k \Delta t}^{(k+1) \Delta t}\left\|P_{N} S_{m \Delta t-s} \partial\right\|_{L(V, V)}\left\|X_{k \Delta t}^{N}(\omega)\right\|_{V}\left\|\left(O_{s}^{N}(\omega)-O_{k \Delta t}^{N}(\omega)\right)\right\|_{V} d s \\
& +\sum_{k=0}^{m-2} \int_{k \Delta t}^{(k+1) \Delta t}\left\|P_{N} S_{m \Delta t-s} \partial\right\|_{L(V, V)} \cdot\left\|\left(O_{s}^{N}(\omega)-O_{k \Delta t}^{N}(\omega)\right)^{2}\right\|_{V} d s \\
& \leq 2 R^{2}(\omega) \sum_{k=0}^{m-2} \int_{k \Delta t}^{(k+1) \Delta t}(m \Delta t-(k+1) \Delta t)^{-\frac{3}{4}} \cdot(s-k \Delta t)^{\theta} d s \\
& +R^{2}(\omega) \sum_{k=0}^{m-2} \int_{k \Delta t}^{(k+1) \Delta t}(m \Delta t-(k+1) \Delta t)^{-\frac{3}{4}}(s-k \Delta t)^{2 \theta} d s \\
& \leq C(R(\omega), \theta)(\Delta t)^{\theta},
\end{aligned}
$$

where the constant depends on $R$ and $\theta$.

Finally, for the third term in (28) again by using this fact that $S_{t}$ is the 
semigroup generated by Laplacian, we have

$$
\begin{aligned}
& \left\|\sum_{k=0}^{m-2} \int_{k \Delta t}^{(k+1) \Delta t}\left(P_{N} S_{m \Delta t-s}-P_{N} S_{m \Delta t-k \Delta t}\right) F\left(X_{k \Delta t}^{N}(\omega)\right) d s\right\|_{V} \\
& \leq \sum_{k=0}^{m-2} \int_{k \Delta t}^{(k+1) \Delta t}\left\|P_{N} S_{m \Delta t-k \Delta t}\left(S_{k \Delta t-s}-I\right) F\left(X_{k \Delta t}^{N}(\omega)\right)\right\|_{V} d s \\
& \leq \sum_{k=0}^{m-2} \int_{k \Delta t}^{(k+1) \Delta t}(m \Delta t-k \Delta t)^{-1}(k \Delta t-s)\left\|F\left(X_{k \Delta t}^{N}(\omega)\right)\right\|_{W} d s \\
& \leq C(R(\omega), T) \Delta t
\end{aligned}
$$

where we used $\left\|P_{N} \Delta S_{t}\right\|_{L(W, V)} \leq C t^{-1}$, together with $\left\|\Delta^{-1}\left(S_{t}-I\right)\right\|_{L(W, V)} \leq t$. Hence from (29) and (30) we derive

$$
\left\|X_{m \Delta t}^{N}(\omega)-Y_{m}^{N}(\omega)\right\|_{V} \leq C\left(R(\omega), R^{2}(\omega), \theta, T\right)(\Delta t)^{\min \left\{\frac{1}{4}, \theta\right\}} .
$$

Let us now turn to the second error term in (26). Note that $Y_{m}^{N, M}: \Omega \rightarrow V$ satisfies

$$
Y_{m}^{N, M}(\omega)=\sum_{k=0}^{m-1} \int_{k \Delta t}^{(k+1) \Delta t} P_{N} S_{m \Delta t-k \Delta t} F\left(Y_{k}^{N, M}(\omega)\right) d s+P_{N} O_{m \Delta t}(\omega) .
$$

Thus by using $\left\|P_{N} S_{t} \partial\right\|_{L(V, V)} \leq C t^{-\frac{3}{4}}$, we can estimate

$$
\begin{aligned}
& \left\|Y_{m}^{N}-Y_{m}^{N, M}\right\|_{V}=\left\|\sum_{k=0}^{m-1} \int_{k \Delta t}^{(k+1) \Delta t} P_{N} S_{m \Delta t-k \Delta t}\left(F\left(X_{k \Delta t}^{N}\right)-F\left(Y_{k}^{N, M}\right)\right)\right\|_{V} \\
& \leq \sum_{k=0}^{m-1} \int_{k \Delta t}^{(k+1) \Delta t}(m \Delta t-k \Delta t)^{-\frac{3}{4}}\left\|\left(X_{k \Delta t}^{N}-Y_{k}^{N, M}\right)^{2}+2 X_{k \Delta t}^{N}\left(X_{k \Delta t}^{N}-Y_{k}^{N, M}\right)\right\|_{V} d s \\
& \leq \sum_{k=0}^{m-1} \Delta t(m \Delta t-k \Delta t)^{-\frac{3}{4}}\left(\left\|X_{k \Delta t}^{N}-Y_{k}^{N, M}\right\|_{V}^{2}+2 R(\omega)\left\|X_{k \Delta t}^{N}-Y_{k}^{N, M}\right\|_{V}\right) .
\end{aligned}
$$

Combining (31) with (33), we have

$$
\begin{aligned}
& \left\|X_{m \Delta t}^{N}(\omega)-Y_{m}^{N, M}(\omega)\right\|_{V} \leq C(R(\omega), \theta, T)(\Delta t)^{\min \left\{\frac{1}{4}, \theta\right\}} \\
& \quad+\sum_{k=0}^{m-1}\left\|X_{k \Delta t}^{N}(\omega)-Y_{k}^{N, M}(\omega)\right\|_{V}^{2}+2 R(\omega) \sum_{k=0}^{m-1}\left\|X_{k \Delta t}^{N}(\omega)-Y_{k}^{N, M}(\omega)\right\|_{V} .
\end{aligned}
$$

If we assume that for some $\delta>0$ fixed later

$$
\sup _{0 \leq k \leq M}\left\|X_{k \Delta t}^{N}(\omega)-Y_{k}^{N, M}(\omega)\right\|_{V} \leq \delta,
$$

then

$$
\begin{aligned}
\left\|X_{m \Delta t}^{N}(\omega)-Y_{m}^{N, M}(\omega)\right\|_{V} & \leq C(R(\omega), \theta, T)(\Delta t)^{\min \left\{\frac{1}{4}, \theta\right\}} \\
& +(\delta+2 R(\omega)) \sum_{k=0}^{m-1}\left\|X_{k \Delta t}^{N}(\omega)-Y_{k}^{N, M}(\omega)\right\|_{V} .
\end{aligned}
$$


Then by the discrete Gronwall Lemma we can conclude

$$
\left\|X_{m \Delta t}^{N}(\omega)-Y_{m}^{N, M}(\omega)\right\|_{V} \leq e^{(m-1)(\delta+2 R(\omega))} C(R(\omega), \theta, T)(\Delta t)^{\min \left\{\frac{1}{4}, \theta\right\}} .
$$

In order to verify (35) we need

$$
e^{(m-1)(\delta+2 R)} C(R(\omega), \theta, T)(\Delta t)^{\min \left\{\frac{1}{4}, \theta\right\}} \leq \delta,
$$

which is true for any $\delta>0$ provided $\Delta t$ is sufficiently small. This finishes the proof of the time discretization.

From Theorem 1 for the spatial discretization error we verified in Section 3

$$
\left\|X_{m \Delta t}(\omega)-X_{m \Delta t}^{N}(\omega)\right\|_{V} \leq C(\omega) \cdot N^{-\gamma},
$$

and from Theorem 11 for the temporal discretization error we just established

$$
\left\|X_{m \Delta t}^{N}(\omega)-Y_{m}^{N, M}(\omega)\right\|_{V} \leq C(R(\omega), \theta, T)(\Delta t)^{\min \left\{\frac{1}{4}, \theta\right\}} .
$$

Therefore we have proved the following Theorem for the stochastic Burgers equation.

Theorem 12. Assume $\rho>0$ such that

$$
\sum_{i, j \in N}\|i\|_{2}^{\rho-1}\|j\|_{2}^{\rho-1}\left|\left\langle Q e_{i}, e_{j}\right\rangle\right|<\infty
$$

Let $X:[0, T] \times \Omega \rightarrow V$ be the solution of SPDE (7) and $Y_{m}^{N, M}: \Omega \rightarrow V$, $m \in\{0,1, \ldots, M\}, M, N \in \mathbb{N}$ the numerical solution given by (22). Fix $\theta \in$ $\left(0, \min \left\{\frac{1}{2}, \frac{\rho}{2}\right\}\right)$ and $\gamma \in\left[0, \frac{1}{2}\right)$.

Then there exists a finite random variable $C: \Omega \rightarrow[0, \infty)$ such that

$$
\left\|X_{m \Delta t}(\omega)-Y_{m}^{N, M}(\omega)\right\|_{V} \leq C(\omega)\left(N^{-\gamma}+(\Delta t)^{\min \left\{\frac{1}{4}, \theta\right\}}\right)
$$

for all $m \in\{0,1, \ldots, M\}$ and every $M, N \in \mathbb{N}$.

\section{$5 \quad$ Numerical results}

In this section we consider the numerical solution of stochastic Burgers equation by the method given in (22).

Consider the stochastic evolution equation (7) with $S:(0, T] \rightarrow L(W, V)$, $F: V \rightarrow W$ given by Lemma 2, Lemma 3 for $T=1, d=1$, and some initial condition fixed to be $(\xi(\omega))(x)=\frac{6}{5} \sin (x)$, for all $x \in[0, \pi]$.

We assume that $O:[0, T] \times \Omega \rightarrow V$ is given by Lemma 4 where the Brownian motion $\beta^{i}:[0, T] \times \Omega \rightarrow \mathbb{R}, i \in \mathbb{N}^{d}$, are dependent by the relation

$$
\mathbb{E}\left(\beta^{k} \beta^{l}\right)=<Q e_{k}, e_{l}>, \quad k, l \in \mathbb{N},
$$

where the covariance operator $Q$ is explicitly given as a convolution operator

$$
<Q e_{k}, e_{l}>=\int_{0}^{\pi} \int_{0}^{\pi} e_{k}(x) e_{l}(y) q(x-y) d y d x,
$$


with kernel

$$
q(x-y)=\max \left\{0, \frac{h-|x-y|}{h^{2}}\right\}
$$

where we define the orthonormal basis

$$
e_{k}(x)=\sqrt{\frac{2}{\pi}} \sin (k x), \quad \text { for } k \in \mathbb{N} .
$$

The possibly small quantity $h>0$ measures the correlation length of the noise. In this case the covariance matrix, i.e., $\left\langle Q e_{k}, e_{l}\right\rangle_{k, l}$, is not diagonal. But for small $h>0$ it is close to diagonal. In Figure 1, the covariance matrix is plotted for $k, l \in\{1,2, \cdots, 100\}$ for $h=0.1,0.01$. Then by some numerical calculations we can show that the condition on $Q$ from (21) is satisfied for any $\rho \in\left(0, \frac{1}{2}\right)$.

The stochastic evolution equation (7) reduces to

$$
d X_{t}=\left[\frac{\partial^{2}}{\partial x^{2}} X_{t}-X_{t} \cdot \frac{\partial}{\partial x} X_{t}\right] d t+d W_{t}, \quad X_{0}(x)=\frac{6}{5} \sin (x)
$$

with $X_{t}(0)=X_{t}(\pi)=0$ for $t \in[0,1]$ and $x \in[0, \pi]$.

The finite dimensional $\operatorname{SDE}(6)$ reduces to

$$
d X_{t}^{N}=\left[\frac{\partial^{2}}{\partial x^{2}} X_{t}^{N}-P_{N}\left(X_{t}^{N} \cdot \frac{\partial}{\partial x} X_{t}^{N}\right)\right] d t+P_{N} d W_{t}, \quad X_{0}^{N}(x)=\frac{6}{5} \sin (x),
$$

with $X_{t}^{N}(0)=X_{t}^{N}(\pi)=0$ for $t \in[0,1]$ and $x \in[0, \pi]$, and all $N \in \mathbb{N}$.

In Figure 2, $O:[0, T] \times \Omega \rightarrow C^{0}([0, \pi])$, the solution of the linear SPDE

$$
d O_{t}=\Delta O_{t} d t+d W_{t},\left.\quad O_{t}\right|_{\partial(0, \pi)}=0, \quad O_{0}=\frac{6}{5} \sin (x),
$$

for $T=1$ is plotted.

Theorem 12 yields the existence of a unique solution $X:[0, \pi] \times \Omega \rightarrow$ $C^{0}([0, \pi])$ of the SPDE (43) such that

$$
\sup _{0 \leq x \leq \pi}\left|X_{m \Delta t}(\omega, x)-Y_{m}^{N, M}(\omega, x)\right| \leq C(\omega)\left(N^{-\gamma}+(\Delta t)^{\min \left\{\frac{1}{4}, \theta\right\}}\right)
$$

for $m=1, \ldots, M, \quad M=\frac{1}{\Delta t}$, such that $\gamma \in\left(0, \frac{1}{2}\right), \theta \in\left(0, \frac{1}{4}\right)$.

By using $\Delta t=\frac{T}{N^{2}}$, the solutions $X_{t}^{N}(\omega, x)$ of the finite dimensional SODEs (44) converge uniformly in $t \in[0,1]$ and $x \in[0, \pi]$ to the solution $X_{t}(\omega, x)$ of the stochastic Burgers equation (43) with the rate $\frac{1}{2}$, as $N$ goes to infinity for all $\omega \in \Omega$. In Figure 3 the pathwise approximation error

$$
\sup _{0 \leq x \leq \pi} \sup _{0 \leq m \leq M}\left|X_{m \Delta t}(\omega, x)-Y_{m}^{N, M}(\omega, x)\right|
$$

is plotted against $N$, for $N \in\{16,32, \cdots, 256\}$. As a replacement for the unknown solution, we use a numerical approximation for $N$ sufficiently large.

Figure 3 confirms that, as we expected from Theorem 12, the order of convergence is $\frac{1}{2}$. Obviously, these are only two examples, but all of a few hundred calculated examples behave similarly. Even their mean seem to behave with the same order of the error. Nevertheless, we did not prove this here and also did not calculate the mean with sufficiently good standard deviation.

Finally, as an example in Figure $4, X_{t}(\omega), x \in[0, \pi]$, is plotted for $t \in$ $\left\{0, \frac{3}{200}, 0.2,1\right\}$, for $h=0.01,0.1$. 


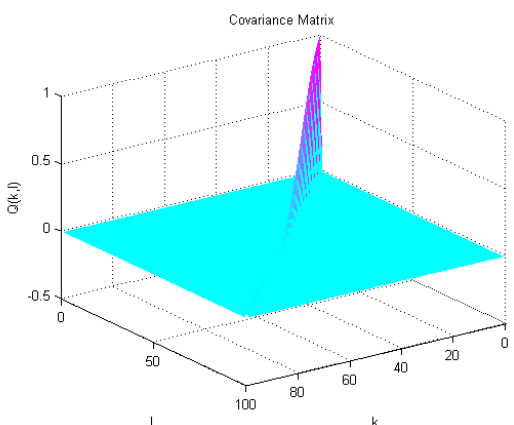

(a)

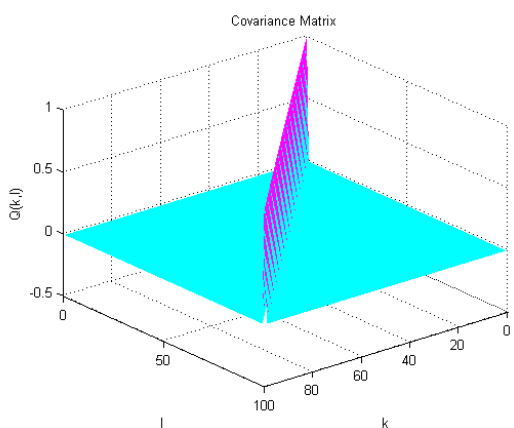

(b)

Figure 1: Covariance Matrix $<Q e_{k}, e_{l}>_{k, l}$ for $k, l \in\{1,2, \cdots, 100\}$, for (a) $h=0.1$ and (b) $h=0.01$.

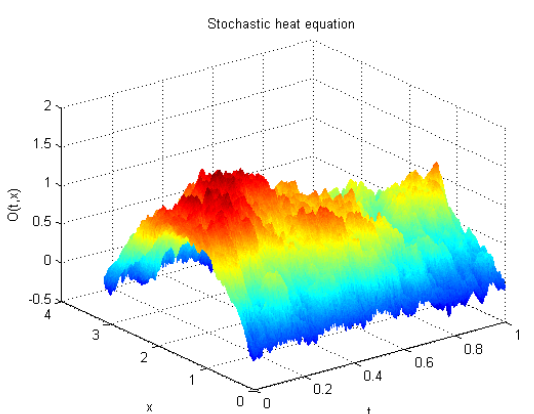

(a)

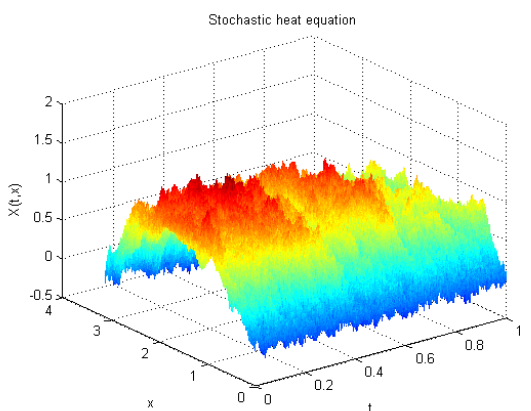

(b)

Figure 2: $O_{t}(\omega, x), x \in[0, \pi], t \in[0,1]$ and one random $\omega \in \Omega$, for (a) $h=0.1$ and (b) $h=0.01$.

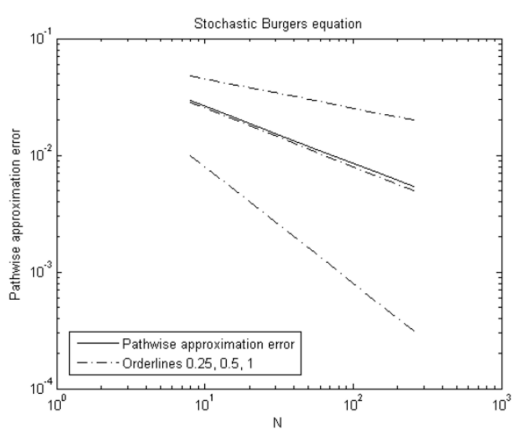

(a)

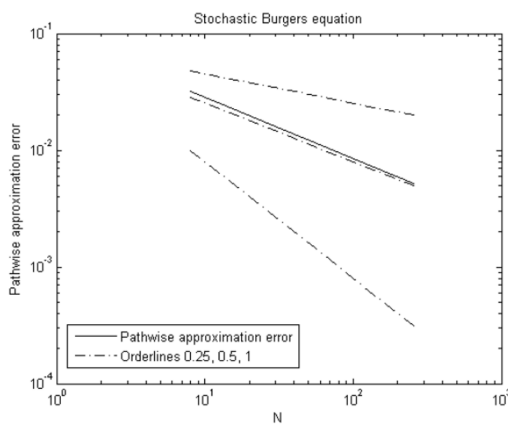

(b)

Figure 3: Pathwise approximation error (46) against $N$ for $N \in\{16,32, \ldots, 256\}$ for two random $\omega \in \Omega$, with $h=0.1$. These are only two examples, but all other calculated trajectories behave similarly. 

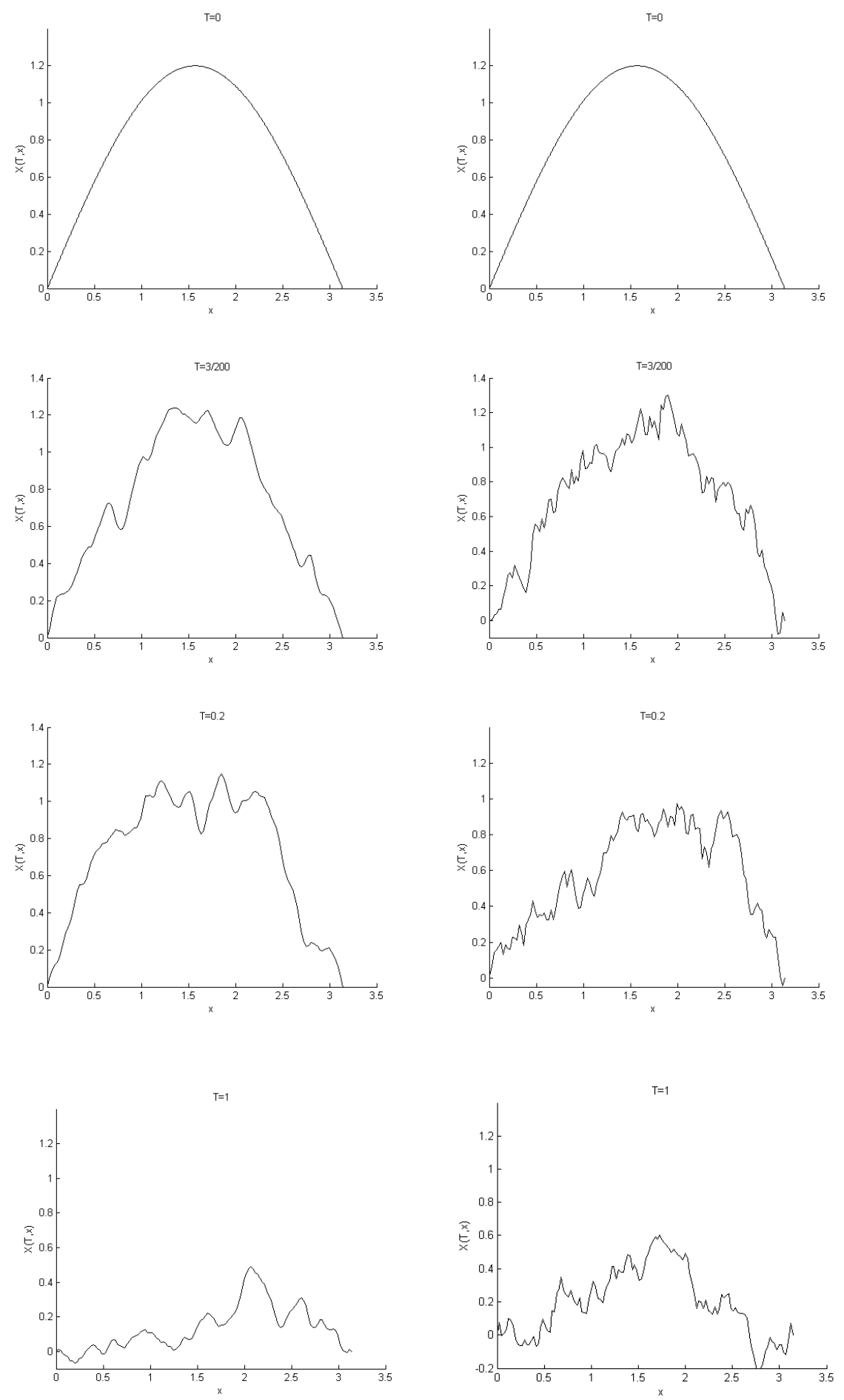

(a)

(b)

Figure 4: Stochastic Burgers equation $X_{t}(\omega, x), x \in[0, \pi], t \in\{0,3 / 200,0.2,1\}$, given by (43) for (a) $h=0.1$ and (b) $h=0.01$, for one random $\omega \in \Omega$. 


\section{Acknowledgment}

The authors of Tarbiat Modares University would like to thank the Department of Mathematics of University of Augsburg for its supports during the second author's visit and also for providing an opportunity of joint research collaborations.

\section{References}

[1] A. Alabert, I. Gyöngy, On numerical approximation of stochastic Burgers equation, From stochastic calculus to mathematical finance, Springer, Berlin, 2006, pp. 1-15.

[2] D. Blömker, J. Duan, Predictability of the Burgers dynamics under model uncertainty. pp. 71-89 in Stochastic differential equations: theory and applications. A volume in honor of Professor Boris L. Rozovskii. P. Baxendale, (ed.) et al., Hackensack, NJ: World Scientific. Interdisciplinary Mathematical Sciences 2,(2007).

[3] D. Blömker, A. Jentzen, Galerkin Approximations for the Stochastic Burgers Equation, (2009), preprint.

[4] G. Da Prato, A. Debussche, R. Temam. Stochastic Burgers equation. Nonlinear Diff. Equ. Appl. 1 (1994), 389-402.

[5] G. Da Prato, D. Gatarek, Stochastic Burgers equation with correlated noise, Stochastics Rep. 52, 1-2 (1995), 29-41.

[6] G. Da Prato, J. Zabczyk, Stochastic equations in infinite dimensions, vol. 44 of Encyclopedia of of Mathematics and its Applications. Cambridge University Press, Cambridge, 1992.

[7] G. Da Prato, J. Zabczyk, Ergodicity for infinite-dimensional systems, vol. 229 of London Mathematical Society Lecture Note Series. Cambridge University Press, Cambridge, 1996.

[8] B. Goldys, B. Maslowski, Exponential ergodicity for stochastic Burgers and $2 D$ Navier-Stokes equations. J. Funct. Anal. 226(1):230-255, (2005).

[9] I. Gyöngy, D. Nualart, On the stochastic Burgers equation in the real line, Ann. Probab. 27, 2 (1999), 782-802.

[10] I. Gyöngy, Lattice approximations for stochastic quasi-linear parabolic partial differential equations driven by space-time white noise II, Potential Anal. 11, (1999), 1-37.

[11] E. Hausenblas, Numerical analysis of semilinear stochastic evolution equations in Banach spaces, J. Comput. Appl. Math. 147(2), (2002), 485-516.

[12] E. Hausenblas, Approximation for Semilinear Stochastic Evolution Equations, Potential Anal. 18(2), (2003), 141-186. 
[13] A. Jentzen, Pathwise Numerical Approximations of SPDEs with Additive Noise under Non-global Lipschitz Coefficients, Potential Anal. 31, (2009), 375-404.

[14] A. Jentzen, P. Kloeden, A. Neuenkirch, Pathwise approximation of stochastic differential equations on domains: higher order convergence rates without global Lipschitz coefficients, Numer. Math. 112(1), (2009), 41-64.

[15] A. Jentzen, P. Kloeden, G. Winkel, Efficient simulation of nonlinear parabolic Spdes with additive noise, Annals of Applied Probability. 21(3), (2011), 908-950.

[16] P.E. Kloeden, S. Shott, Linear-implicit strong schemes for Itô-Galerkin approximations of stochastic PDEs, J. Appl. Math. Stochastic Anal. 14(1), (2001), 47-53. Special issue: Advances in applied stochastics.

[17] Di Liu, Convergence of the spectral method for stochastic Ginzburg equation driven by space-time white noise, Comm. Math. Sci, 1(2), (2003), 361-375.

[18] G. Lord, J. Rougemont, A Numerical Scheme for Stochastic PDEs with Gevrey Regularity, IMA J. Num. Anal, 24, (2004), 587-604.

[19] G. Lord, T. Shardlow, Postprocessing for stochastic parabolic partial differential equations, SIAM J. Numer. Anal. 45(2), (2007), 870-889.

[20] T. Runst, W. Sickel, Sobolev spaces of fractional order, Nemytskij operators, and nonlinear partial differential equations, vol. 3 of de Gruyter Series in Nonlinear Analysis and Applications. Walter de Gruyter Co., Berlin, 1996.

[21] T. Shardlow, Numerical methods for stochastic parabolic PDEs, Numer. Funct. Anal. Optim. 20, (1999), 121-145.

[22] H. Yoo, Semi-discretization of stochastic partial differential equations on $R$ by a finite difference method, Mathematics of computation, 69, (1999), 653-666. 